\title{
Johannes Görbert/Nikolas Immer (Hg.): Ambulante Poesie. Explorationen deutschsprachiger Reiselyrik seit dem 18. Jahrhundert
}

Stuttgart: J.B. Metzler 2020 - ISBN 978-3-476-05116-5 - 84,99 €.

https://doi.org/10.14361/zig-2021-120115

Gewiss besteht (nicht allein) die deutschsprachige Reiseliteratur vorwiegend aus umfangreichen Prosatexten, die mit ausführlichen Beschreibungen mehr oder minder fremder Land- und Völkerschaften aufwarten. Allerdings ist auch die durch Verknappung und Verdichtung gekennzeichnete Gattung der Lyrik in diesem Zusammenhang nicht ohne Belang, existiert doch eine durchaus beträchtliche Anzahl einschlägiger Werke. So verweisen die Herausgeber des hier $\mathrm{zu}$ besprechenden Bandes, Johannes Görbert und Nikolas Immer, in ihrer Einleitung auf Gedichte bzw. Gedichtzyklen von Goethe und Hölderlin, Tieck und Heine, Benn und Enzensberger sowie auf manch weitere, meist weniger kanonische Beispiele. Angesichts dessen wirkt es unbefriedigend, dass sich die seit den 1970er Jahren enorm angewachsene Reiseliteratur-Forschung der Lyrik nur selten zugewandt hat. Somit widmen sich die von Görbert und Immer versammelten Studien, die mehrheitlich auf im Rahmen einer Berner Konferenz gehaltenen Vorträgen basieren, einem echten Desiderat.

Unklar bleibt freilich, weshalb die Aufsätze nicht das gesamte historische Spektrum der Neueren deutschen Literaturwissenschaft abdecken, sondern lediglich, wie im Untertitel vermerkt, den Zeitraum vom 18. bis zum 21. Jahrhundert. Denn ausweislich ihrer Einleitung ist Görbert und Immer vollends bewusst, dass etwa auch die Reisegedichte Paul Flemings, der in den 1630er Jahren bis nach Persien gelangt war, Be- rücksichtigung verdient hätten (vgl. $3 \mathrm{u}$. 8f.). Darüber hinaus wäre eingehender $\mathrm{zu}$ diskutieren gewesen, inwiefern die Lyrik der vom Hitler-Regime ins Exil gezwungenen Autorinnen und Autoren tatsächlich der Reiseliteratur zugeordnet werden kann bzw. sollte (vgl. 6), zumal es diesbezüglich ja eine bedeutende eigenständige Forschungstradition gibt. Dagegen leuchtet der Vorschlag der Herausgeber, in typologischer Absicht zwischen faktualen, fiktionalen und reflexiven Reisegedichten $\mathrm{zu}$ unterscheiden, unmittelbar ein. Letztere verzichten ihnen zufolge auf die »Gestaltung der Durchquerung von Räumen durch eine lyrische Sprechinstanz« (8) und verhandeln die Reisethematik stattdessen auf einer Metaebene. (Indes irritiert es, dass das Adjektiv sfaktuak in diesem Kontext einige Male unpassend gebraucht wird; an den betreffenden Stellen müsste es sreak heißen.)

Besonders ergiebig fällt die Einleitung also dort aus, wo Görbert und Immer die zunächst gewählte quantitative Perspektive auf die Reiselyrik um eine qualitative ergänzen: Nicht nur eine möglichst große Materialmenge wollen sie erschließen, sondern auch die spezifischen Verfahren und Funktionen von Gedichten innerhalb der Reiseliteratur. Für elementar erachten sie dabei »die Aspekte der Subjektivierung, Pointierung und Emotionalisierung « (10), die sie denn auch anschaulich erläutern. Es dürfte jedoch kaum zu leugnen sein, dass diese Aspekte in epischen Texten ebenfalls zum Tragen kommen (können). 
Wichtig erscheint sodann Görberts und Immers Hinweis auf die hohe Zahl intertextueller Referenzen, die sich in etlichen Reisegedichten findet (vgl. 11). Vor allem aber betonen die Herausgeber, dass viele lyrische Formtraditionen in bestimmten Weltregionen geprägt wurden; als Beispiele dienen ihnen unter anderem »de[r] griechisch-römische[ ] Hexameter « und »die romanischen Strophenformen Terzine, Ritornell und Stanze« (11). Analog dazu verhalte es sich so, »dass Reisedichter wie etwa Goethe, Heine und Durs Grünbein bei ihren jeweiligen Aufenthalten in Italien, im Harz und in Tokyo auf korrespondierende lyrische Formen wie auf das Epigramm, auf den volksliedhaften Vierzeiler bzw. auf das Haiku zurückgreifen.« (12)

Nun ist es für Sammelbände keineswegs untypisch, dass die Kerngedanken der Einleitung in den einzelnen Beiträgen allenfalls sporadisch aufgegriffen werden. Der Aufsatz von Ralph Müller aber läuft den Überlegungen Görberts und Immers sogar partiell zuwider, vertritt doch sein Autor die Auffassung, dass ein Gedicht allein dann zur Reiseliteratur zu zählen sei, wenn es »in den Kontext eines minimalen (vermutlich faktischen) Reisenarrativs gestellt werden kann und [...] als eine Auseinandersetzung mit einer persönlichen Erfahrung von Fremdem und Anderem an einem anderen Ort erscheint « (21) - was auf die von den Herausgebern erwähnten reflexiven Reisegedichte mitnichten zutrifft. Unabhängig davon präsentiert Müller ein beachtliches Panorama der deutschsprachigen Reiselyrik von Goethes 1790 entstandenen Venezianischen Epigrammen bis zu Barbara Köhlers Gedichtband Istanbul, zusehends von 2015. Außerdem gerät sein Befund, dass den Werken des Genres oft »eine starke Tendenz zum Epigrammatischen« (41) eigne, durchaus plausibel.
Den Auftakt zum wenig aussagekräftig betitelten Abschnitt »Kulturelle Konstruktionen « bildet Christopher Meids erhellender Beitrag über Goethes Römische Elegien (1795), die ihm zufolge eine »Kombination von Bildungs- und Vergnügungsreise« (47) schildern. Dabei resultiere der Anschein der Authentizität paradoxerweise just aus dem Umstand, dass Goethe beständig auf die Literatur der Antike rekurriert. Erörtert wird demnach der Konnex von Intertextualität und Interkulturalität, wie er auch für Dominik Zinks Studie zu Tiecks Reisegedichten eines Kranken und Rückkehr eines Gesunden (1821/23) von eminenter Relevanz ist. Mit seinem Italien-Zyklus knüpfe Tieck lose »an die Tradition der Reiseliteratur der Aufklärung« an, wobei es ihm »die lyrische Form « gestatte, »die interkulturelle Situation in ihrer Faktizität zu ihrem Recht kommen zu lassen, sie aber gleichzeitig in ihrer Kontingenz und Veränderbarkeit zu begreifen.«(62)

Anschließend nimmt Nikolas Immer die von Levin Schücking zusammengestellte Anthologie Italia. Deutsche Dichter als Führer jenseits der Alpen (1851) in den Blick, ehe sich Alexander Quack dem Zyklus Südliche Landschaft (1947) von Marie Luise Kaschnitz zuwendet. Sein Augenmerk richtet er unter anderem auf die in den Gedichten entworfene Relation von Vergangenheit und Gegenwart, und mithin beschäftigt er sich nicht nur mit der für die Reiseliteratur zentralen Dimension des Raums, sondern auch mit derjenigen der Zeit. Dieses Vorgehen mutet zweifellos produktiv an, doch tritt mitunter ein fragwürdiges Literaturkonzept zutage, etwa wenn Quack einen Teilvers Kaschnitz' "als dunkle Prophezeiung « (109) verstanden wissen will. Yvonne Nilges wiederum muss weit ausholen und um mehrere Ecken denken, um Ingeborg Bachmanns Italiengedichte überhaupt als reiseliterarische Texte klassifizieren 
zu können: Zuflucht sucht sie schließlich beim Begriff der »abstrakte[n] Reiselyrik« (119; Hervorh. i.O.). Dessen ungeachtet vermag Nilges zu zeigen, mittels welcher Strategien Bachmanns Gedichte konstitutive Elemente des Selbstbildes der österreichischen und der deutschen Nachkriegsgesellschaft unterminieren.

Während sämtliche Aufsätze der Sektion "Kulturelle Konstruktionen" Werke mit Italienbezug analysieren, setzen sich die Beiträge der gleichermaßen unpräzise benannten Abteilung »Spatiale Explorationen« mit Texten über ganz unterschiedliche Erdgegenden auseinander. So behandelt die eher thesenarme, aber informative Studie von Sonja Klimek jene Gedichte der Frühaufklärerin Sidonia Hedwig Zäunemann, die deren Reiseerlebnisse in Thüringen thematisieren. Der komparatistische Aufsatz von Siegfried Ulbrecht vergleicht Heines Zyklus Aus der Harzreise (1827) mit Puškins um 1830 entstandener Kaukasus-Lyrik, wohingegen sich Stefani Kuglers luzide Untersuchung mit Lenaus exotistischen USA-Gedichten aus den 1830er Jahren befasst. In diesen werde die indigene Bevölkerung $\mathrm{zu}$ naturverbundenen sedlen Wilden stilisiert, die aufgrund der "genozidalen Umsiedlungspolitik« der Regierung dem Untergang geweiht sind: "Indem die Balladen den hegemonialen Diskurs ungebrochen poetisch überhöhen«, so Kugler, »vollziehen sie die Exklusionspolitik auf der rhetorisch-ästhetischen Ebene mit.«(205)

Der Beitrag von Olga Bezantakou rekonstruiert die Entwicklung der deutschsprachigen Athen-Lyrik zwischen 1838 und 1935 und führt im Zuge dessen vor Augen, auf welche Weise der literarische Philhellenismus seine rein klassizistische Ausrichtung allmählich hinter sich ließ. Daraufhin präsentiert Evelyn Dueck eine Vielzahl lohnender Beobachtungen zu Hilde Domins 1964 erschienener Gedichtsammlung Hier, und zwar insbesondere zum sich darin entfaltenden Sprach- und Literaturverständnis. Allerdings wird, wie bereits in Görberts und Immers Einleitung, nicht recht ersichtlich, was denn eigentlich dafür spricht, Gedichte über Flucht und Exil der Reiseliteratur zuzurechnen. Ratsam wäre eine intensivere Beschäftigung mit dieser Frage schon deshalb gewesen, weil Domin, wie Dueck selbst hervorhebt, wiederholt eine strikte Unterscheidung zwischen Vertreibungs- und Reiseerfahrungen angemahnt hat (vgl. 224f.).

Den Schlussabschnitt "Generische Transgressionen« eröffnet Sarah Thierys ebenso material- wie kenntnisreicher Aufsatz über die Lyrisierung von Eisenbahnreisen durch Autorinnen und Autoren der Neuen Sachlichkeit, darunter Kurt Tucholsky, Erich Kästner und Mascha Kaléko. Es folgt Julia Ilgners ungemein gründliche, aber auch mit Detailinformationen überfrachtete Interpretation von Richard Dehmels Epigrammzyklus Eine Rundreise in Ansichtspostkarten (1906): Da dessen Anlage entscheidend vom im Titel genannten Bild-Text-Medium beeinflusst sei, stelle er ein hochgradig innovatives Formexperiment dar. Der Beitrag von Bernhard Metz verlässt sodann den Gegenstandsbereich der Germanistik und fokussiert die Verschränkung von Reise- und Flaneriediskurs in Michèle Métails französischsprachigen Berlin-Gedichten, die auf prominente Vorgängertexte wie die von Franz Hessel oder Walter Benjamin referieren. Im Anschluss charakterisiert Johannes Görbert die Haikus aus Durs Grünbeins Band Lob des Taifuns (2008) unter anderem als anti-exotistische Kunstwerke, die eine "allgemeinmenschliche [...] Perspektive auf Japan« vermitteln bzw. "anthropologische Konstanten« (337) zum Vorschein bringen sollen. Hier wäre wohl kritisch zu fragen 
gewesen, inwieweit Grünbeins lyrischer Universalismus der Vereinnahmung des Fremden und der Nivellierung seiner Alterität Vorschub leistet, doch ändert dies nichts daran, dass man Görberts Aufsatz mit großem Gewinn liest. Letzteres gilt auch für denjenigen von Ingo Irsigler, der sich mit Mondreisen-Schilderungen in Popsongs von David Bowie, Elton John und Peter Schilling sowie in Gedichten von Gerhard Rühm und Durs Grünbein befasst. In allen Fällen erzeuge die Mondreise »eine Distanzierung, die [...] kulturelle Reflexionen ermöglichen soll«, etwa »über die Grenzen der Kunst « oder »die Bestimmung des Poetischen« (361).
$\mathrm{Zu}$ bilanzieren bleibt, dass Görbert und Immer einen instruktiven (und sorgfältig lektorierten) Band vorgelegt haben, der für die Reiseliteratur-Forschung von einigem Wert ist. Selbstredend erscheinen manche Beiträge einschlägiger als andere, und selbstredend variiert die Qualität der Studien merklich. In ihrer Gesamtheit aber erweitern die Aufsätze den Kenntnisstand zur deutschsprachigen Reiselyrik in erheblichem Maße und das ist kein geringes Verdienst.

Stefan Hermes

(http://orcid.org/0000-0001-7487-3945) 